Trinity University

Digital Commons @ Trinity

Mathematics Faculty Research

Mathematics Department

$12-2011$

\title{
Bifurcation and Invariant Manifolds of the Logistic Competition Model
}

M. Guzowska

Rafael Luís

Saber Elaydi

Trinity University, selaydi@trinity.edu

Follow this and additional works at: https://digitalcommons.trinity.edu/math_faculty

Part of the Mathematics Commons

\section{Repository Citation}

Guzowska, M., Luís, R., \& Elyadi, S. (2011). Bifurcation and invariant manifolds of the logistic competition model. Journal of Difference Equations and Applications, 17(12), 1851-1872. http://doi.org/10.1080/ 10236198.2010.504377

This Post-Print is brought to you for free and open access by the Mathematics Department at Digital Commons @ Trinity. It has been accepted for inclusion in Mathematics Faculty Research by an authorized administrator of Digital Commons @ Trinity. For more information, please contact jcostanz@trinity.edu. 
Journal of Difference Equations and Applications

Vol. 00, No. 00, May 2010, 1-21

\title{
Bifurcation and invariant manifolds of the logistic competition model
}

\author{
Malgorzata Guzowska ${ }^{\mathrm{a}}$, Rafael Luís ${ }^{\mathrm{b}}{ }^{\dagger}$, and Saber Elaydic ${ }^{\mathrm{c}}$ \\ ${ }^{a}$ Department of Econometrics and Statistics, University of Szczecin, Szczecin, Poland; \\ ${ }^{\mathrm{b}}$ Center for Mathematical Analysis, Geometry, and Dynamical Systems, Instituto

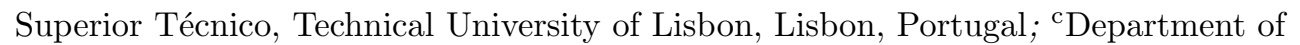 \\ Mathematics, Trinity University, San Antonio, Texas, USA.
}

(Received)

\begin{abstract}
In this paper we study a new logistic competition model. We will investigate stability and bifurcation of the model. In particular, we compute the invariant manifolds, including the important center manifolds, and study their bifurcation. Saddle-node and period doubling bifurcation route to chaos is exhibited via numerical simulations.
\end{abstract}

Keywords: Stability, Bifurcation, Competition Model, Center Manifolds, Unstable and Stable Manifolds.

\section{Introduction}

There are many discrete competition models applied to biology and economics in the literature, and we cite a few here $[3,4,6-9,13-15,17,19,20]$. However, we are going to develop a new competition model based on sound biological assumptions of intra-specific and inter-specific competitions.

In developing this model, it is assumed that, without interspecific competition, each species is modeled by the logistic map. The logistic map is used to model species with non overlapping generations under the assumption that the fitness function decreases when the population density (size) increases. Let $z_{n}$ be the density of species $z$ at time period $n$. Then the fitness function is defined as $u(z)=\frac{z_{n+1}}{z_{n}}$. When the population is sufficiently small (close to zero), the intraspecific competition (competition among individuals of species $z$ ) is negligible and consequently, $u(z)=\frac{z_{n+1}}{z_{n}}=R$, where $R>1$ is a constant, commonly called, the intrinsic growth rate of the population. When the population grows, the fitness function decreases due to significant intraspecific competition and reaches the value 1 when the population density reaches the carrying capacity $K$.

Figure 1 depicts a typical fitness function. Assuming that the decrease in the fitness function is linear, then the model is obtained by finding the equation of the line connecting the points $(0, R)$ and $(K, 1)$. The equation of this line is given by

$$
\frac{z_{n+1}}{z_{n}}=-\frac{R-1}{K} z_{n}+R
$$

\footnotetext{
๑Email: MGuzowska@wneiz.pl

†Corresponding author. Email: rafael.luis.madeira@gmail.com

$\S$ Email: saber.elaydi@trinity.edu
} 


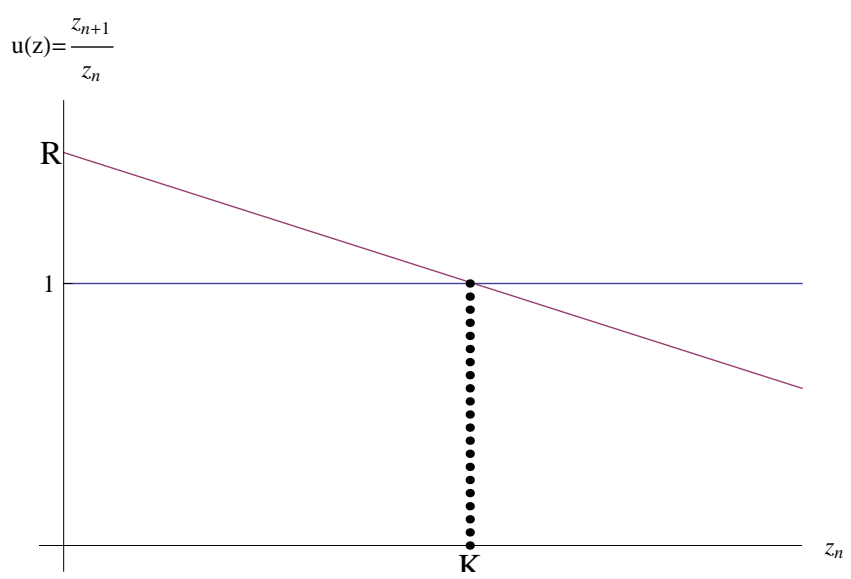

Figure 1. The fitness function

where $R>1$. Using the change of variables, $x_{n}=\frac{R-1}{R K} z_{n}$ we obtain the celebrated logistic difference equation $x_{n+1}=R x_{n}\left(1-x_{n}\right)$.

Introducing a new species $y$ to compete with species $x$, interspecific competition (competition between two different species) would negatively affect the growth of species $x$ and vice-versa. We propose the following new competition model

$$
\left\{\begin{array}{l}
x_{n+1}=\frac{a x_{n}\left(1-x_{n}\right)}{1+c y_{n}} \\
y_{n+1}=\frac{b y_{n}\left(1-y_{n}\right)}{1+d x_{n}}
\end{array},\right.
$$

where $a, b>0$ and $c, d \in(0,1)$. The map associated with equation (1) is given by

$$
F(x, y)=\left(\frac{a x(1-x)}{1+c y}, \frac{b y(1-y)}{1+d x}\right)
$$

To insure that the range of this map lies in the first quadrant, we make the following two assumptions:

(1) $x$ and $y$ are in $[0,1]$,

(2) $a$ and $b$ are in $(0,4]^{1}$.

These two assumptions guarantee that nonnegative points are mapped to nonnegative points and specifically the map $F$ maps $[0,1] \times[0,1]$ into $[0,1] \times[0,1]$. To show this we note that the maximum of the $x$-component of the image of the point $(x, y)$ is $a / 4$ and occurs at $x=1 / 2$ and $y=0$ and the maximum of the $y$-component of the image of the point $(x, y)$ is $b / 4$ and occurs at $x=0$ and $y=1 / 2$.

In model (1) the parameters $a$ and $b$ are known as the intrinsic growth rates of species $x$ and $y$, respectively, and the parameters $c$ and $d$ are known as the competition parameters of species $y$ and $x$, respectively.

The map $F$ has one extinction fixed point $(0,0)$, two exclusion fixed points $\left(\frac{a-1}{a}, 0\right),\left(0, \frac{b-1}{b}\right)$, and one coexistence fixed point

$$
\left(x^{*}, y^{*}\right)=\left(\frac{-c b+a b-b+c}{a b-c d}, \frac{-d a+a b-a+d}{a b-c d}\right) .
$$

In the next result we give sufficient conditions for the stability of the extinction fixed point.

\footnotetext{
${ }^{1}$ In section 5 we will indicate how this restriction on the values of $a$ and $b$ may be slightly relaxed
} 
LEMma 1.1. Let $\left(x_{n}, y_{n}\right)$ denote the solution of the Logistic competition model (1) with an initial condition $\left(x_{0}, y_{0}\right) \in(0,1) \times(0,1)$. If $a \in(0,1]$ then $\lim _{n \rightarrow \infty} x_{n}=0$. If $b \in(0,1]$ then $\lim _{n \rightarrow \infty} y_{n}=0$. Moreover, if $a, b \in(0,1]$, then $\lim _{n \rightarrow \infty}\left(x_{n}, y_{n}\right)=(0,0)$.

Proof. The inequality $x_{n+1} \leq a x_{n}, \forall n \in \mathbb{Z}^{+}$holds since

$$
0 \leq x_{n+1}=\frac{a x_{n}\left(1-x_{n}\right)}{1+c y_{n}} \leq a x_{n}-a x_{n}^{2} \leq a x_{n} .
$$

Let $a \in(0,1)$. Then an induction shows that $x_{n} \leq x_{0} a^{n}$ for all $n \in \mathbb{Z}^{+}$. Thus $\lim _{n \rightarrow \infty} x_{n}=0$.

When $a=1$ one has $x_{n+1}<x_{n}, \forall n \in \mathbb{Z}^{+}$. Thus $x_{n}$ is a decreasing sequence of numbers that is bounded above by 1 and bounded bellow by 0 which implies the convergence of $x_{n}$ as $n$ goes to infinity. Let $L$ be this limit. Then $0 \leq L \leq x_{n}<1$, $\forall n \in \mathbb{Z}^{+}$. Note that from $x_{n+1} \leq x_{n}\left(1-x_{n}\right), \forall n \in \mathbb{Z}^{+}$it follows that

$$
x_{n} \leq x_{0} \prod_{i=0}^{n-1}\left(1-x_{i}\right), \forall n \geq 1 .
$$

By the fact that $1-x_{i} \leq 1-x_{i+1}, i \in \mathbb{Z}^{+}$, induction shows that

$$
\prod_{i=0}^{n-1}\left(1-x_{i}\right) \leq\left(1-x_{n-1}\right)^{n}, \forall n \geq 1 \text {. }
$$

Using this last relation yields

$$
x_{n} \leq \frac{x_{0}}{1-x_{n}} \prod_{i=0}^{n}\left(1-x_{i}\right) \leq \frac{x_{0}}{1-x_{n}}\left(1-x_{n}\right)^{n+1}=\left(1-x_{n}\right)^{n} .
$$

But the relation $L \leq x_{n}<1$ implies that $0<1-x_{n} \leq 1-L, \forall n \in \mathbb{Z}^{+}$and consequently one has

$$
x_{n} \leq\left(1-x_{n}\right)^{n} \leq(1-L)^{n} \underset{n \rightarrow \infty}{\longrightarrow} 0 .
$$

A similar argument proves the assertion when $b \in(0,1]$.

\section{Invariant Manifolds}

Let $F: \mathbb{R}^{k} \rightarrow \mathbb{R}^{k}$ be a map such that $F \in C^{2}$ and $F(0)=0$. Then one may write $F$ as a perturbation of a linear map $L$,

$$
F(X)=L X+R(X)
$$

where $L$ is a $k \times k$ matrix defined by $L=D(F(0)), R(0)=0$ and $D R(0)=0$, where $D$ denotes the derivative. Now we will introduce special subspaces of $\mathbb{R}^{k}$, called invariant manifolds [22], that will play a central role in our study of stability and bifurcation.

An invariant manifolds is a manifold embedded in its phase space with the property that it is invariant under the dynamical system generated by $F$. A subspace 
$M$ of $\mathbb{R}^{k}$ is an invariant manifold if whenever $X \in M$, then $F^{n}(X) \in M$, for all $n \in \mathbb{Z}^{+}$. For the linear map $L$, one may split its spectrum $\sigma(L)$ into three sets $\sigma_{s}$, $\sigma_{u}$, and $\sigma_{c}$, for which $\lambda \in \sigma_{s}$ if $|\lambda|<1, \lambda \in \sigma_{u}$ if $|\lambda|>1$, and $\lambda \in \sigma_{c}$ if $|\lambda|=1$.

Corresponding to these sets, we have three invariant manifolds (linear subspaces) $E^{s}, E^{u}$, and $E^{c}$ which are the generalized eigenspaces corresponding to $\sigma_{s}, \sigma_{u}$, and $\sigma_{c}$, respectively. It should be noted that some of this subspaces may be trivial subspaces.

The main question here is how to extend this linear theory to nonlinear maps. Corresponding to the linear subspaces $E^{s}, E^{u}$, and $E^{c}$, we will have the invariant manifolds the stable manifold $W^{s}$, the unstable manifold $W^{u}$, and the center manifolds $W^{c}$.

The center manifolds theory $[1,2,12,16,21,22]$ is interesting only if $W^{u}=\{0\}$. For in this case, the dynamics on the center manifold $W^{c}$ determines the dynamics of the system. The other interesting case is when $W^{c}=\{0\}$ and we have a saddle.

Let $E^{s} \subset \mathbb{R}^{s}, E^{u} \subset \mathbb{R}^{u}$, and $E^{c} \subset \mathbb{R}^{t}$, with $s+u+t=k$. Then one may formally define the above mentioned invariant manifolds as follows:

$$
\begin{gathered}
W^{s}=\left\{x \in \mathbb{R}^{k} \mid F^{n}(x) \rightarrow 0 \text { as } n \rightarrow \infty\right\} \text { and } \\
W^{u}=\left\{x \in \mathbb{R}^{k} \mid F^{n}(x) \rightarrow 0 \text { as } n \rightarrow-\infty\right\} .
\end{gathered}
$$

Since the stability on the center manifold is not apriori known, we will define it as a manifold of dimension $t$ whose graph is tangent to $E^{c}$ at the origin. It is noteworthy to mention that the center manifold is not unique, while the stable and unstable manifolds are unique.

The next result summarize the basic invariant manifolds theory

Theorem 2.1 Invariant manifolds theorem. [11, 16] Suppose that $F \in C^{2}$. Then there exist $C^{2}$ stable $W^{s}$ and unstable $W^{u}$ manifolds tangent to $E^{s}$ and $E^{u}$, respectively, at $X=0$ and $C^{1}$ center manifold $W^{c}$ tangent to $E^{c}$ at $X=0$. Moreover, the manifolds $W^{c}, W^{s}$ and $W^{u}$ are all invariant.

\subsection{Center manifolds}

In this section, we focus on the case when $\sigma_{u}=\emptyset$. Hence the eigenvalues of $L$ are either inside the unit disk or on the unit disk. By suitable change of variables, one may represent the map $F$ as a system of difference equation such as

$$
\left\{\begin{array}{l}
x_{n+1}=A x_{n}+f\left(x_{n}, y_{n}\right) \\
y_{n+1}=B y_{n}+g\left(x_{n}, y_{n}\right)
\end{array} .\right.
$$

First we assume that all eigenvalues of $A_{t \times t}$ are on the unit circle and all the eigenvalues of $B_{s \times s}$ are inside the unit circle, with $t+s=k$. Moreover,

$$
f(0,0)=0, g(0,0)=0, D f(0,0)=0 \text { and } D g(0,0)=0 .
$$

Since $W^{c}$ is tangent to $E^{c}=\left\{(x, y) \in \mathbb{R}^{t} \times \mathbb{R}^{s} \mid y=0\right\}$, it may be represented locally as the graph of a function $h: \mathbb{R}^{t} \rightarrow \mathbb{R}^{t}$ such that

$$
\begin{gathered}
W^{c}=\left\{(x, y) \in \mathbb{R}^{t} \times \mathbb{R}^{s}|y=h(x), h(0)=0, D h(0)=0,| x \mid<\right. \\
\delta \text { for a sufficiently small } \delta\} .
\end{gathered}
$$

Furthermore, the dynamics restricted to $W^{c}$ is given locally by the equations

$$
x_{n+1}=A x_{n}+f\left(x_{n}, h\left(x_{n}\right)\right), x \in R^{t}
$$



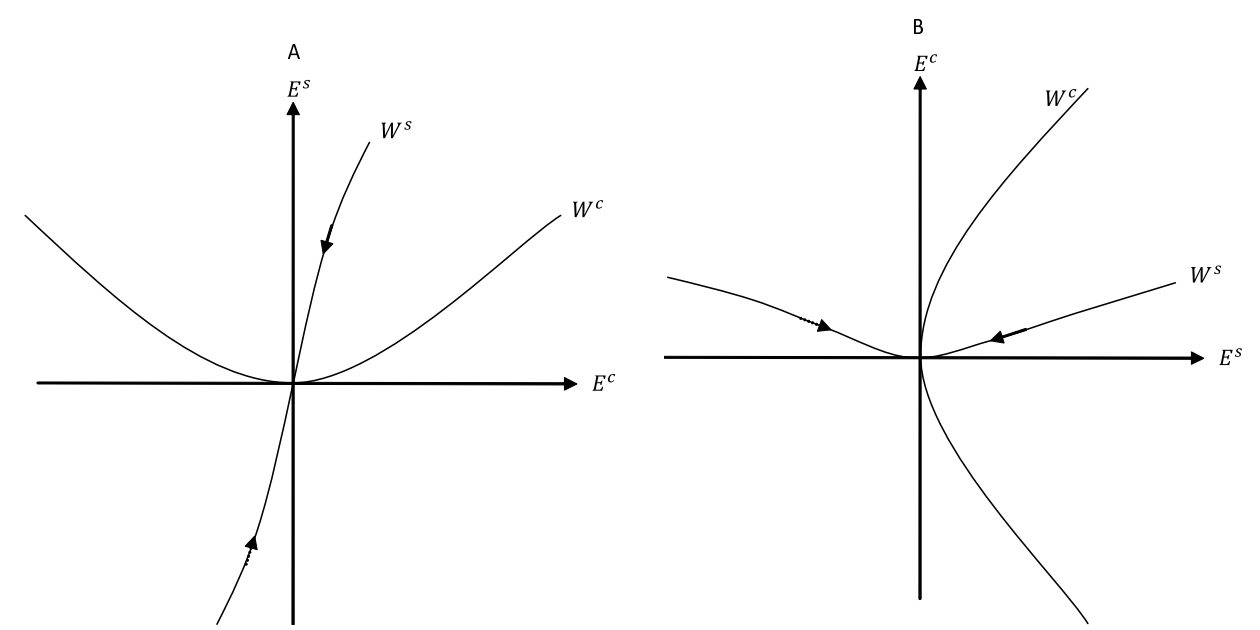

Figure 2. Stable and center manifolds. In Figure A one has $\sigma(A)=\sigma_{c}$ and $\sigma(B)=\sigma_{s}$ while in Figure B one has $\sigma(A)=\sigma_{s}$ and $\sigma(B)=\sigma_{c}$.

The main feature of equation (4) is that its dynamics determine the dynamics of Equation (3). So if $x^{*}=0$ is a stable, asymptotically stable, or unstable fixed point of Eq. (4), then the fixed point $\left(x^{*}, y^{*}\right)=(0,0)$ of Equation $(3)$ possesses the corresponding property.

To find the map $y=h(x)$, we substitute for $y$ in Eq. (3) and obtain

$$
\left\{\begin{array}{l}
x_{n+1}=A x_{n}+f\left(x_{n}, h\left(x_{n}\right)\right) \\
y_{n+1}=h\left(x_{n+1}\right)=h\left(A x_{n}+f\left(x_{n}, h\left(x_{n}\right)\right)\right)
\end{array} .\right.
$$

But

$$
\begin{aligned}
y_{n+1} & =B y_{n}+g\left(x_{n}, y_{n}\right) \\
& =B h\left(x_{n}\right)+g\left(x_{n}, h\left(x_{n}\right)\right) .
\end{aligned}
$$

Equating (5) and (6) yields the center manifold equation

$$
h\left[A x_{n}+f\left(x_{n}, h\left(x_{n}\right)\right)\right]=B h\left(x_{n}\right)+g\left(x_{n}, h\left(x_{n}\right)\right)
$$

Analogously if $\sigma(A)=\sigma_{s}$ and $\sigma(B)=\sigma_{c}$, one may define the center manifold $W^{c}$, and obtain the equation

$$
y_{n+1}=B y_{n}+g\left(h\left(y_{n}\right), y_{n}\right)
$$

where $x=h(y)$.

\subsection{An upper (lower) triangular System}

In working with concrete maps, it is beneficial in certain cases to deal with the system without diagonalization.

Let us now consider the case when $L$ is a block upper triangular matrix

$$
\left(\begin{array}{l}
x_{n+1} \\
y_{n+1}
\end{array}\right)=\left(\begin{array}{ll}
A & C \\
0 & B
\end{array}\right)\left(\begin{array}{l}
x_{n} \\
y_{n}
\end{array}\right)+\left(\begin{array}{l}
f\left(x_{n}, y_{n}\right) \\
g\left(x_{n}, y_{n}\right)
\end{array}\right)
$$


There are two cases to consider:

(1) Assume that $\sigma(A)=\sigma_{s}, \sigma(B)=\sigma_{c}$, and $\sigma_{u}=\emptyset$.

The matrix $L$ can be block diagonalizable. Hence there exists, a nonsingular matrix $P$ of the form

$$
P=\left[\begin{array}{ll}
P_{1} & P_{3} \\
0 & P_{2}
\end{array}\right]
$$

such that

$$
\left[\begin{array}{ll}
A & B \\
0 & C
\end{array}\right]=P\left[\begin{array}{ll}
A & 0 \\
0 & B
\end{array}\right] P^{-1}
$$

Let

$$
\left(\begin{array}{l}
x \\
y
\end{array}\right)=P\left(\begin{array}{l}
u \\
v
\end{array}\right)
$$

Then $x=P_{1} u+P_{3} v$, and $y=P_{2} v$. Thus one has

$$
\left(\begin{array}{l}
u_{n+1} \\
v_{n+1}
\end{array}\right)=\left(\begin{array}{cc}
A & 0 \\
0 & B
\end{array}\right)\left(\begin{array}{l}
u_{n} \\
v_{n}
\end{array}\right)+P^{-1}\left(\begin{array}{c}
f\left(P_{1} u+P_{3} v, P_{2} v\right) \\
g\left(P_{1} u+P_{3} v, P_{2} v\right)
\end{array}\right)
$$

Applying the center manifold theorem to Equation (10) yields a map $u=\tilde{h}(v)$ with $\tilde{h}(0)=0=\tilde{h}^{\prime}(0)$. Moreover, the dynamics of Equations (10) is completely determined by the dynamics of the equation

$$
v_{n+1}=B v_{n}+\tilde{P}_{2} g\left(P_{1} \tilde{h}\left(v_{n}\right)+P_{3} v_{n}, P_{2} v_{n}\right),
$$

where $\tilde{P}_{1}$ and $\tilde{P}_{3}$ are elements of the matrix

$$
P^{-1}=\left[\begin{array}{cc}
\tilde{P}_{1} & \tilde{P}_{3} \\
0 & \tilde{P}_{2}
\end{array}\right]
$$

We now have the relation

$$
u=\tilde{P}_{1} x-\tilde{P}_{2} P_{3} \tilde{P}_{2} y=\tilde{h}\left(\tilde{P}_{2} y\right) .
$$

Hence $x=h(y)$, where $h$ is given by

$$
h(y)=P_{3} \tilde{P}_{2} y+\tilde{P}_{1}^{-1} \tilde{h}_{2}\left(\tilde{P}_{2} y\right)
$$

Notice that $D h(0)=P_{3} \tilde{P}_{2} I$, where $I$ is the identity matrix.

(2) Assume that $\sigma(A)=\sigma_{c}, \sigma(B)=\sigma_{s}$, and $\sigma_{u}=\emptyset$. We start from equation (10) and apply the center manifold theorem to obtain a map $v=\tilde{h}(u)$ with $\tilde{h}(0)=0=\tilde{h}^{\prime}(0)$. The dynamics of equation (10) is completely determined by the dynamics of the equation

$$
u_{n+1}=A u_{n}+\tilde{P}_{1} f\left(P_{1} u_{n}+P_{3} \tilde{h}(u), P_{2} \tilde{h}(u)\right)+\tilde{P}_{3} g\left(P_{1} u_{n}+P_{3} \tilde{h}(u), P_{2} \tilde{h}(u)\right),
$$

where $\tilde{P}_{1}, \tilde{P}_{2}$, and $\tilde{P}_{3}$ are entries of the matrix

$$
P^{-1}=\left(\begin{array}{cc}
\tilde{P}_{1} & \tilde{P}_{3} \\
0 & \tilde{P}_{2}
\end{array}\right)
$$




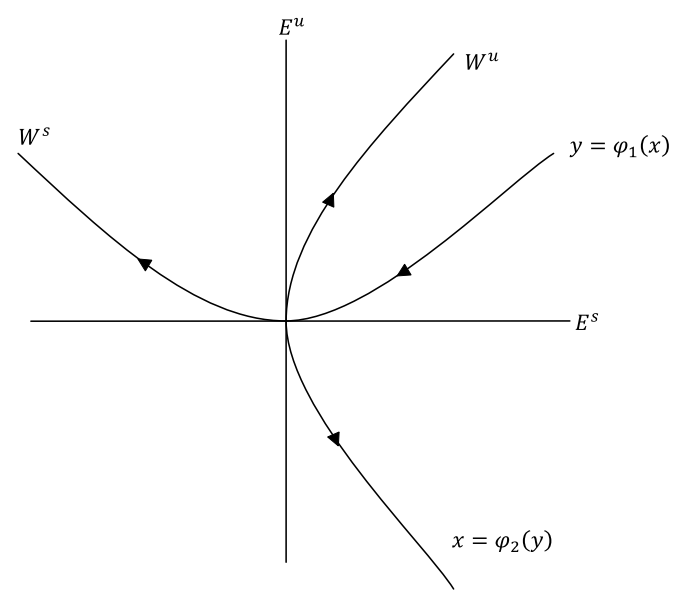

Figure 3. Stable and unstable manifolds

From (9) we have $u=\tilde{P}_{1} x-\tilde{P}_{1} P_{3} \tilde{P}_{2} y$ and $v=\tilde{P}_{2} y$. Then $v=\tilde{h}(u)$ and thus

$$
\tilde{P}_{2} y=\tilde{h}\left(\tilde{P}_{1} x-\tilde{P}_{1} P_{3} \tilde{P}_{2} y\right)
$$

Let $Q(x, y)=\tilde{P}_{2} y-\tilde{h}\left(\tilde{P}_{1} x-\tilde{P}_{1} P_{3} \tilde{P}_{2} y\right)$. Then $Q(0,0)=0, D Q(0,0)$ is of rank $t$. Hence by the implicit function theorem [18] there exits an open neighborhood $\Omega \subset \mathbb{R}^{k}$ of 0 and a unique function $h \in C^{1}(\Omega)$ such that $h(0)=0=D h(0)$ and $Q(x, h(x))=0$, for all $x \in \Omega$.

Hence the curve $y=h(x)$ is the implicit solution of Equation (11) and is the equation of the center manifold. To find the map $h$ we use the center manifold equation

$$
h[A x+C h(x)+f(x, h(x))]=B h(x)+g(x, h(x)) .
$$

A final remark is in order. If we let $y=h(x)$ in (11) we obtain

$$
h(x)=P_{2} \tilde{h}\left(\tilde{P}_{1} x-\tilde{P}_{1} P_{3} \tilde{P}_{2} h(x)\right) .
$$

Note that $\operatorname{Dh}(0)=0=D \tilde{h}(0)$.

\subsection{Stable and Unstable Manifolds}

Suppose now that the map $F$ is hyperbolic, that is $\sigma_{c}=\emptyset$. Then by theorem 2.1, there are two unique invariant manifolds $W^{s}$ and $W^{u}$ tangents to $E^{s}$ and $E^{u}$ at $X=0$, which are graphs of the maps

$$
\varphi_{1}: E_{1} \rightarrow E_{2} \text { and } \varphi_{2}: E_{2} \rightarrow E_{1},
$$

such that

$$
\varphi_{1}(0)=\varphi_{2}(0)=0 \text { and } D\left(\varphi_{1}(0)\right)=D\left(\varphi_{2}(0)\right)=0 .
$$

Letting $y_{n}=\varphi_{1}\left(x_{n}\right)$ yields

$$
y_{n+1}=\varphi_{1}\left(x_{n+1}\right)=\varphi_{1}\left(A x_{n}+f\left(x_{n}, \varphi_{1}\left(x_{n}\right)\right)\right) .
$$


But

$$
y_{n+1}=B \varphi_{1}\left(x_{n}\right)+g\left(x_{n}, \varphi_{1}\left(x_{n}\right)\right) .
$$

Equating these two equations yields

$$
\varphi_{1}\left(A x_{n}+C \varphi_{1}\left(x_{n}\right)+f\left(x_{n}, \varphi_{1}(x)\right)\right)=B \varphi_{1}\left(x_{n}\right)+g\left(x_{n}, \varphi_{1}\left(x_{n}\right)\right)
$$

where we can take, without loss of generality, $\varphi_{1}(x)=\alpha_{1} x^{2}+\beta_{1} x^{3}+O\left(|x|^{4}\right)$.

Similarly, letting $x_{n}=\varphi_{2}\left(y_{n}\right)$ yields

$$
x_{n+1}=\varphi_{2}\left(y_{n+1}\right)=\varphi_{2}\left(B y_{n}+g\left(\varphi_{2}\left(y_{n}\right), y_{n}\right)\right),
$$

where we can take, without loss of generality, $\varphi_{2}(x)=\alpha_{2} x+\beta_{2} x^{2}+O\left(|x|^{4}\right)$

But

$$
x_{n+1}=A \varphi_{2}\left(y_{n}\right)+C y_{n}+f\left(\varphi_{2}\left(y_{n}\right), y_{n}\right)
$$

and hence

$$
\varphi_{2}\left(B y_{n}+g\left(\varphi_{2}\left(y_{n}\right), y_{n}\right)\right)=A \varphi_{2}\left(y_{n}\right)+C y_{n}+f\left(\varphi_{2}\left(y_{n}\right), y_{n}\right)
$$

Using equations (12) and (13), one can find the stable manifold

$$
W^{s}=\left\{(x, y) \in \mathbb{R}^{t} \times \mathbb{R}^{s} \mid y=\phi_{1}(x)\right\},
$$

and the unstable manifold

$$
W^{u}=\left\{(x, y) \in \mathbb{R}^{t} \times \mathbb{R}^{s} \mid x=\phi_{2}(y)\right\}
$$

\section{Stability of the exclusion fixed points}

In this section we investigate the local stability of the exclusion fixed points of the logistic competition model using standard linearization techniques.

The Jacobian of the map $F$ given in (1) is given by

$$
J F(x, y)=\left[\begin{array}{ll}
\frac{a(1-x)-a x}{1+c y} & \frac{-a c x(1-x)}{(1+c y)^{2}} \\
\frac{-b d y(1-y)}{(1+d x)^{2}} & \frac{b(1-y)-b y}{1+d x}
\end{array}\right]
$$

The Jacobian evaluated at the fixed point $(0,0)$ is given by

$$
J_{0}=J F(0,0)=\left[\begin{array}{ll}
a & 0 \\
0 & b
\end{array}\right]
$$

If $a \leq 1$ and $b \leq 1$, then by lemma 1.1 the exclusion fixed point $(0,0)$ is globally asymptotically stable. Clearly, when $a>1$ or $b>1$, the fixed point $(0,0)$ becomes unstable.

Note that if $y_{n}=0$, then $x_{n}$ follows the dynamics of the standard logistic map in which $x_{n} \rightarrow 0$ as $n \rightarrow \infty$ whenever $a \leq 1$. Analogously, $y_{n} \rightarrow 0$ as $n \rightarrow \infty$ if $x_{n}=0$ and $b \leq 1$. 
The Jacobian evaluated at the fixed point $\left(\frac{a-1}{a}, 0\right)$ is given by

$$
J_{a}=J F\left(\frac{a-1}{a}, 0\right)=\left[\begin{array}{ll}
2-a \frac{-c(a-1)}{a} \\
0 & \frac{a b}{a d+a-d}
\end{array}\right] .
$$

The eigenvalues of $J_{a}$ are $\lambda_{1}=2-a$ and $\lambda_{2}=\frac{a b}{a d+a-d}$. Hence the fixed point $\left(\frac{a-1}{a}, 0\right)$ is asymptotically stable if $1<a<3$ and $1<b<1+d \frac{a-1}{a}$. If $1<a<3$ and $b=1$, then $\left|\lambda_{1}\right|<1$ and $\lambda_{2}=\frac{a}{a+d(a-1)}<1$ and consequently $\left(\frac{a-1}{a}, 0\right)$ is asymptotically stable. If $a=1$ we have only the extinction fixed point $(0,0)$. In conclusion, the fixed point $\left(\frac{a-1}{a}, 0\right)$ is asymptotically stable if $1<a<3$ and $1<b<1+d\left(\frac{a-1}{a}\right)$.

Two issues remain unresolved. The first is the case when $a=3$ and $b<1+\frac{d(a-1)}{a}$, in which the eigenvalue $\lambda_{1}=-1$ and $\lambda_{2}<1$. The second case is when $1<a<3$ and $b=1+d\left(d \frac{a-1}{a}\right)$ in which $\left|\lambda_{1}\right|<1$ and $\lambda_{2}=1$. To investigate these cases, we need to use the theory developed in the previous section.

Let us now consider the first case. In order to apply the center manifold theorem, we make a change of variables in system (1) so we can have a shift from the point $\left(\frac{a-1}{a}, 0\right)$ to $(0,0)$.

Let $u=x-\frac{a-1}{a}$ and $v=y$. Then the new system is given by

$$
\left\{\begin{array}{l}
u_{n+1}=\frac{a\left(u_{n}+\frac{a-1}{a}\right)\left(1-\left(u_{n}+\frac{a-1}{a}\right)\right)}{1+c v_{n}}-\frac{a-1}{a} \\
v_{n+1}=\frac{b v_{n}\left(1-v_{n}\right)}{1+d\left(u_{n}+\frac{a-1}{a}\right)}
\end{array} .\right.
$$

The Jacobian of the planar map given in (14) is

$$
\tilde{J} F(u, v)=\left[\begin{array}{cc}
\frac{-(2 a u-2+a)}{1+c v} & \frac{c(a u+a-1)(a u-1)}{a(1+c v)^{2}} \\
\frac{a^{2} b d v(v-1)}{(a+a d u+a d-d)^{2}} & \frac{-a b(2 v-1)}{a+a d u+a d-d}
\end{array}\right] .
$$

At $(0,0), \tilde{J} F$ has the form

$$
\tilde{J}_{0}=\tilde{J} F(0,0)=\left[\begin{array}{lr}
2-a \frac{-c(a-1)}{a} \\
0 & \frac{a b}{a+a d-d}
\end{array}\right] .
$$

When $a=3$ we have

$$
\tilde{J}_{0}=\tilde{J} F(0,0)=\left[\begin{array}{lc}
-1 & \frac{-2 c}{3} \\
0 & \frac{3 b}{3+2 d}
\end{array}\right] .
$$

Now we can write the equations in system (14) as

$$
\left\{\begin{array}{l}
u_{n+1}=-u_{n}-\frac{2}{3} c v_{n}+\tilde{f}\left(u_{n}, v_{n}\right) \\
v_{n+1}=\frac{3 b}{3+2 d} v_{n}+\tilde{g}\left(u_{n}, v_{n}\right)
\end{array},\right.
$$

where 


$$
\tilde{f}\left(u_{n}, v_{n}\right)=\frac{1}{3} \frac{3 c u_{n} v_{n}+2 c^{2} v_{n}^{2}-9 u_{n}^{2}}{1+c v_{n}}
$$

and

$$
\tilde{g}\left(u_{n}, v_{n}\right)=-3 b v_{n} \frac{3 d u_{n}+3 v_{n}+2 d v_{n}}{9+9 d u_{n}+12 d+6 d^{2} u_{n}+4 d^{2}} .
$$

Let us assume that the map $h$ takes the form

$$
h(u)=\alpha u^{2}+\beta u^{3}+O\left(u^{4}\right), \alpha, \beta \in \mathbb{R} .
$$

Now we are going to compute the constants $\alpha$ and $\beta$. The function $h$ must satisfy the center manifold equation

$$
h\left(-u-\frac{2}{3} \operatorname{ch}(u)+\tilde{f}(u, h(u))\right)-\frac{3 b}{3+2 d} h(u)-\tilde{g}(u, h(u))=0
$$

This leads to the following polynomial equation

$$
p_{1} u^{2}+p_{2} u^{3}+\ldots+p_{14} u^{15}=0
$$

where the coefficients $p_{i}, i=1, \ldots, 14$ are in appendix A.

Solving the system $p_{1}=0$ and $p_{2}=0$ yields the unique solution $\alpha=0$ and $\beta=0$. $P_{3}=0$ yields the coefficient of the fourth degree after substituting for $\alpha=0$ and $\beta=0$. Similarly one may show that all the coefficients of the polynomial are equal to zero. Hence $h(u)=0$. Thus on the center manifold $v=0$ we have the following map

$$
Q(u)=-u-3 u^{2}
$$

Simple computations show that the Schwarzian derivative of the map $Q$ at the origin is -54 . Hence, by [5] the exclusion fixed point $(2 / 3,0)$ is asymptotically stable.

Remark 1. One may reach the same conclusion by making few observation based on the well known dynamics of the one-dimensional logistic map [5]. First notice that the positive $u$-axis is invariant under the map $F$. Moreover, the positive $u$-axis is in the same direction as the eigenspace $E^{c}$. Hence the positive $u$-axis is a center manifold $W^{c}$. Second, the fixed point $2 / 3$ is asymptotically stable under the one-dimensional logistic map and thus on the center manifold $W^{c}$ the fixed point $(2 / 3,0)$ is asymptotically stable.

Finally, we investigate the case when $1<a<3$ and $b=1+d\left(\frac{a-1}{a}\right)$. In this case we have $\left|\lambda_{1}\right|<1$ and $\lambda_{2}=1$. When $b=1+d\left(\frac{a-1}{a}\right)$ we have

$$
\tilde{J}_{0}=\tilde{J} F(0,0)=\left[\begin{array}{l}
2-a \frac{-(a-1) c}{a} \\
0
\end{array}\right] .
$$

Now we can write the equations in system (14) as 


$$
\left(\begin{array}{l}
u_{n+1} \\
v_{n+1}
\end{array}\right)=\left(\begin{array}{c}
2-a \frac{-(a-1) c}{a} \\
0 \\
1
\end{array}\right)\left(\begin{array}{l}
u_{n} \\
v_{n}
\end{array}\right)+\left(\begin{array}{l}
\hat{f}\left(u_{n}, v_{n}\right) \\
\hat{g}\left(u_{n}, v_{n}\right)
\end{array}\right)
$$

where

$$
\hat{f}(u, v)=\frac{-a^{2} u^{2}-2 a c u v+a^{2} c u v-c^{2} v^{2}+a c^{2} v^{2}}{a(1+c v)}
$$

and

$$
\hat{g}(u, v)=-\frac{v(-d v+a(v+d(u+v)))}{-d+a(1+d+d u)} .
$$

The eigenvalues of the linear part are $\lambda_{1}=2-a$ and $\lambda_{2}=1$, with corresponding eigenvectors $V_{1}=(1,0)$ and $V_{2}=\left(-\frac{c}{a}, 1\right)$. Let us assume that

$$
u=h(v)=-\frac{c}{a} v+\alpha v^{2}+\beta v^{3} .
$$

The map $h$ must satisfy the equation

$$
h(v+\hat{g}(h(v), v))-(2-a) h(v)+\frac{c(a-1)}{a} v-\hat{f}(h(v), v)=0 .
$$

After some computer manipulations we get the equation

$$
p_{1} v^{2}+p_{2} v^{3}+\ldots+p_{14} v^{15}=0
$$

where

$$
\begin{aligned}
p_{1}= & a^{3} c-3 a^{2} c d+3 a^{3} c d-a^{2} c^{2} d+3 a c d^{2}-6 a^{2} c d^{2}+3 a^{3} c d^{2}+2 a c^{2} d^{2}-2 a^{2} c^{2} d^{2}- \\
& c d^{3}+3 a c d^{3}-3 a^{2} c d^{3}+a^{3} c d^{3}-c^{2} d^{3}+2 a c^{2} d^{3}-a^{2} c^{2} d^{3}-a^{4} \alpha+a^{5} \alpha+3 a^{3} d \alpha- \\
& 6 a^{4} d \alpha+3 a^{5} d \alpha-3 a^{2} d^{2} \alpha+9 a^{3} d^{2} \alpha-9 a^{4} d^{2} \alpha+3 a^{5} d^{2} \alpha+a d^{3} \alpha-4 a^{2} d^{3} \alpha+ \\
& 6 a^{3} d^{3} \alpha-4 a^{4} d^{3} \alpha+a^{5} d^{3} \alpha
\end{aligned}
$$

and

$$
\begin{aligned}
p_{2}= & a^{3} c^{2}-5 a^{2} c^{2} d+3 a^{3} c^{2} d-a^{2} c^{3} d+7 a c^{2} d^{2}-10 a^{2} c^{2} d^{2}+3 a^{3} c^{2} d^{2}+4 a c^{3} d^{2}- \\
& 2 a^{2} c^{3} d^{2}-3 c^{2} d^{3}+7 a c^{2} d^{3}-5 a^{2} c^{2} d^{3}+a^{3} c^{2} d^{3}-3 c^{3} d^{3}+4 a c^{3} d^{3}-a^{2} c^{3} d^{3}- \\
& 2 a^{4} \alpha-a^{4} c \alpha+6 a^{3} d \alpha-6 a^{4} d \alpha+9 a^{3} c d \alpha-6 a^{4} c d \alpha-6 a^{2} d^{2} \alpha+12 a^{3} d^{2} \alpha- \\
& 6 a^{4} d^{2} \alpha-15 a^{2} c d^{2} \alpha+24 a^{3} c d^{2} \alpha-9 a^{4} c d^{2} \alpha+2 a d^{3} \alpha-6 a^{2} d^{3} \alpha+6 a^{3} d^{3} \alpha-2 a^{4} d^{3} \alpha+ \\
& 7 a c d^{3} \alpha-18 a^{2} c d^{3} \alpha+15 a^{3} c d^{3} \alpha-4 a^{4} c d^{3} \alpha-a^{4} \beta+a^{5} \beta+3 a^{3} d \beta-6 a^{4} d \beta+3 a^{5} d \beta- \\
& 3 a^{2} d^{2} \beta+9 a^{3} d^{2} \beta-9 a^{4} d^{2} \beta+3 a^{5} d^{2} \beta+a d^{3} \beta-4 a^{2} d^{3} \beta+6 a^{3} d^{3} \beta-4 a^{4} d^{3} \beta+a^{5} d^{3} \beta .
\end{aligned}
$$

Solving the system $p_{1}=0$ and $p_{2}=0$ we obtain the values

$$
\begin{aligned}
\beta= & -\frac{2 a^{2} c+a^{3} c^{2}-4 a c d+4 a^{2} c d-6 a c^{2} d-a^{2} c^{2} d+2 a^{3} c^{2} d-a^{2} c^{3} d+2 c d^{2}-4 a c d^{2}}{(-1+a)^{2} a(a-d+a d)^{2}} \\
& +\frac{2 a^{2} c d^{2}+6 c^{2} d^{2}-6 a c^{2} d^{2}-a^{2} c^{2} d^{2}+a^{3} c^{2} d^{2}+4 c^{3} d^{2}-a^{2} c^{3} d^{2}}{(-1+a)^{2} a(a-d+a d)^{2}}
\end{aligned}
$$


and

$$
\alpha=-\frac{a c-c d+a c d-c^{2} d}{\left(-a+a^{2}\right)(a-d+a d)}
$$

and consequently, the map $P$ on the center manifolds is given by

$$
P(v)=-\frac{(a-d+a d)(-1+v) v}{-d(1+c v)+a\left(1+d+d v^{2} \alpha+d v^{3} \beta\right)} .
$$

Computations show that $P^{\prime}(0)=1$, and $P^{\prime \prime}(0)=\frac{2((1+c) d-a(1+d))}{a-d+a d} \neq 0$ and thus the exclusion fixed point $\left(\frac{a-1}{a}, 0\right)$ on the center manifold $u=h(v)$ is unstable.

Analogous results may be obtained for the exclusion fixed point $\left(0, \frac{b-1}{b}\right)$.
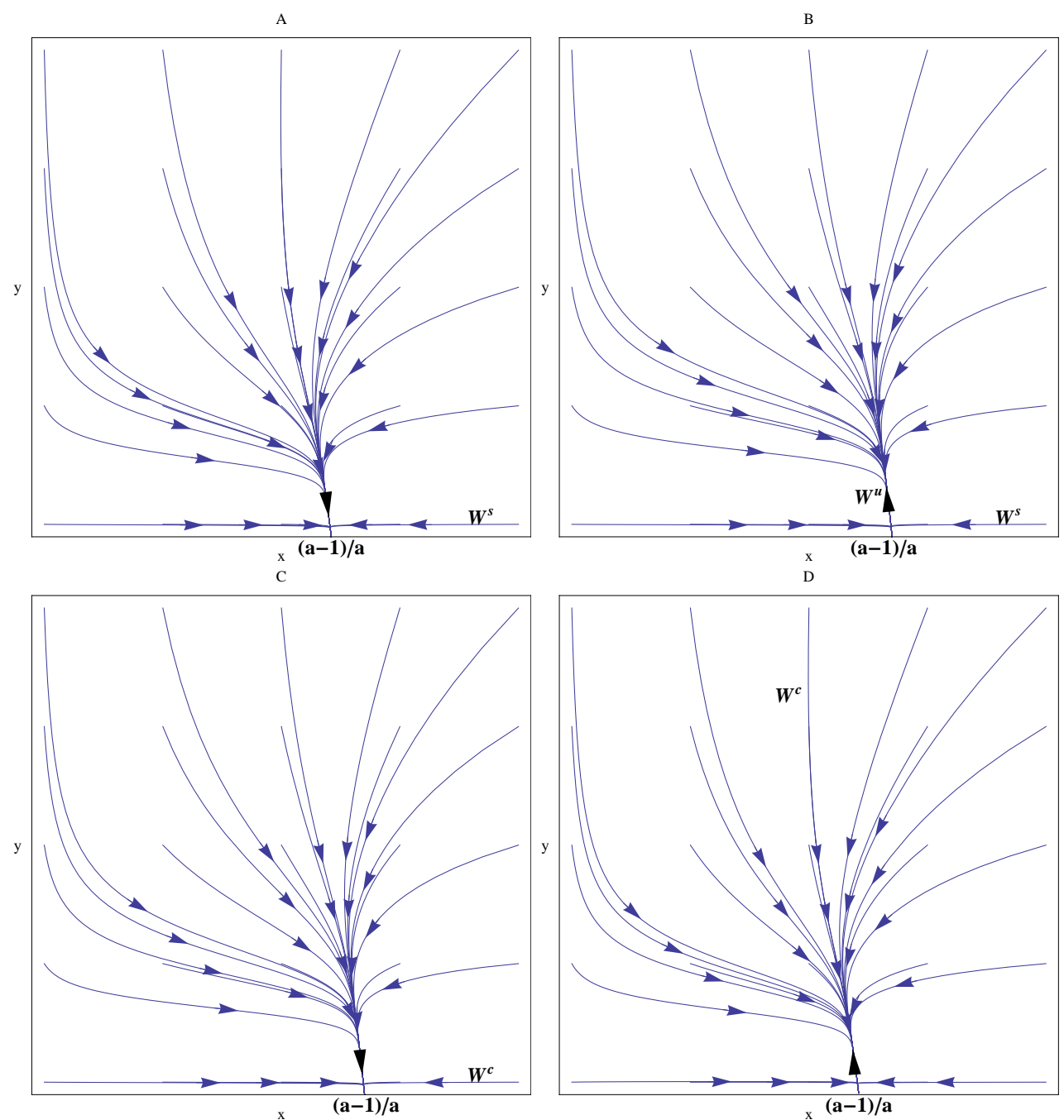

Figure 4. The phase-space diagram for the exclusion fixed point $\left(\frac{a-1}{a}, 0\right)$. A - The exclusion fixed point is asymptotically stable: $a=2.5, b=1.01, c=0.3, d=0.1$. B - The exclusion fixed point is a saddle: $a=2, b=1.1, c=0.3, d=0.1$. C - The exclusion fixed point is a saddle $a=3, b=1.01, c=0.3, d=0.1$, where the center manifold is stable on the $x$-axis. D - The exclusion fixed point is a saddle $a=2.5$, $b=1.06, c=0.3, d=0.1$ where the center manifold is unstable on the $x$-axis and in the interior of the first quadrant

In Figures 4 and 5 we present the phase-space diagram for this exclusion fixed point. In Figure 4A both eigenvalues are inside the unit circle, i.e., $1<a<3$ and $1<b<1+d\left(\frac{a-1}{a}\right)$ and the stable manifold is on the $x$-axis. Figure $4 \mathrm{~B}$ 
shows the unstable and stable manifolds when $1<a<3$ and $b>1+d\left(\frac{a-1}{a}\right)$, i.e., the first eigenvalue is inside the unit circle and the second is outside the unit circle. In Figure $4 \mathrm{C}$ the stable center manifold is on the $x$-axis when $a=3$ and $1<b<1+d\left(\frac{a-1}{a}\right)$. In this case the first eigenvalue is -1 and the second eigenvalue is inside the unit circle. Figure $4 \mathrm{D}$ shows the unstable center manifold when the first eigenvalue is inside the unit circle and the second is on the unit circle. This happens when $1<a<3$ and $b=1+d\left(\frac{a-1}{a}\right)$.

When $a>3$ and $1<b<1+d\left(\frac{a-1}{a}\right)$ the exclusion fixed point $\left(\frac{a-1}{a}, 0\right)$ becomes unstable and $2-$ periodic orbit is created (Figure 5 ).

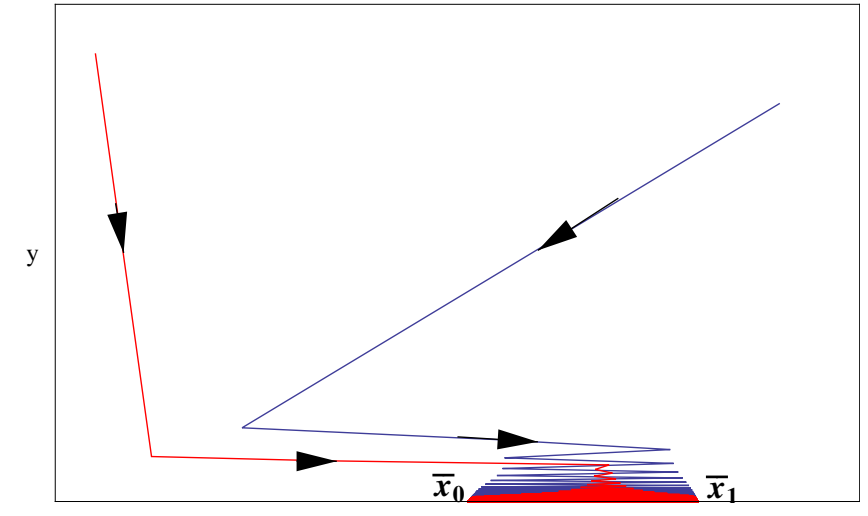

Figure 5. The existence of an exclusion asymptotically stable 2 -periodic cycle on the $x$-axis when one eigenvalue is outside the unit circle and the second eigenvalue is inside the unit circle

Before end this section we note that the exclusion principle in Biology is valid for both species. In Figure 6 is presented the two possible scenarios. In Figure 6A species $y$ goes extinct while in Figure 6B species $x$ goes extinct.

\section{Stability of the coexistence fixed point}

In this section we study the stability of the coexistence fixed point

$$
\left(x^{*}, y^{*}\right)=\left(\frac{-c b+a b-b+c}{a b-c d}, \frac{-d a+a b-a+d}{a b-c d}\right),
$$

which is the solution of the system

$$
\left\{\begin{array}{l}
a x+c y=a-1 \\
d x+b y=b-1
\end{array}\right.
$$

These two lines are called the isoclines of Eq. (1). On the first line $s_{1}: a x+c y=$ $a-1$, the $x$-coordinate of each point is fixed while on the second line $s_{2}: d x+b y=$ $b-1$, the $y$-coordinate of each point is fixed (Figure 7)

In the literature these line segments are known as isoclines. It helps in the existence and in the stability of the equilibria for the species. A specie's isocline indicates the combination of $x^{*}$ and $y^{*}$ for which the population has no net growth, i.e, $x_{n+1} / x_{n}=1$ and $y_{n+1} / y_{n}=1$.

The existence of the coexistence fixed point $\left(x^{*}, y^{*}\right)$ is insured by the following conditions

$$
\frac{a-1}{c}>\frac{b-1}{b} \text { and } \frac{b-1}{d}>\frac{a-1}{a}
$$



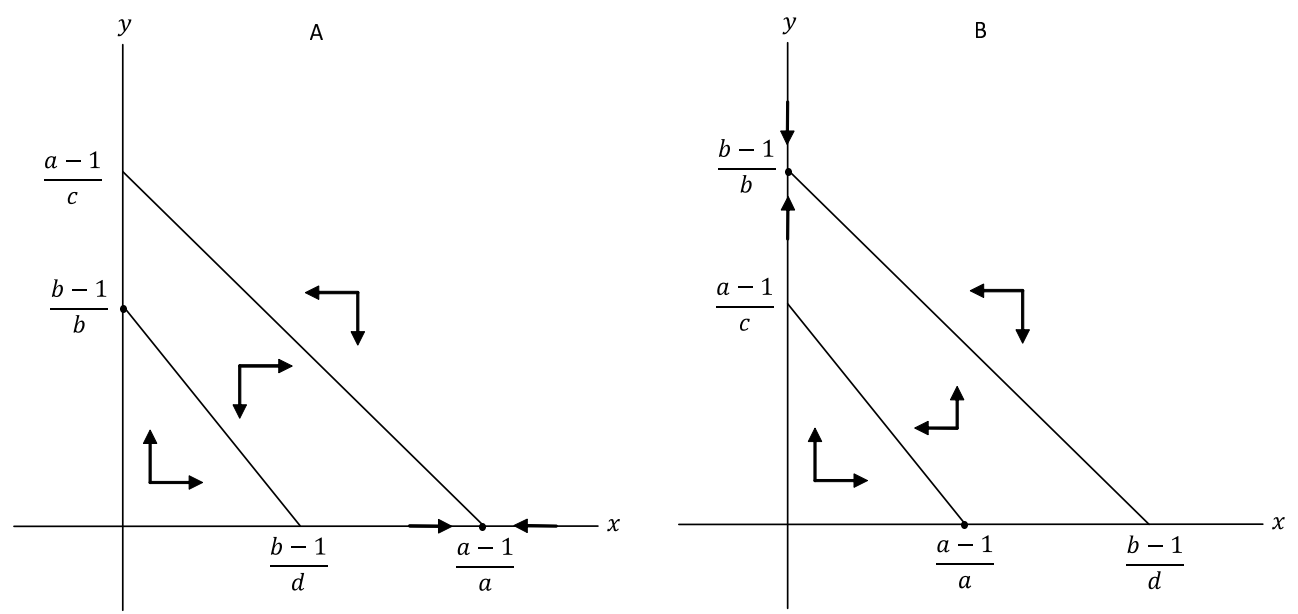

Figure 6. The stability of the exclusion fixed points and the validity of the exclusion principle. A - If $1<a \leq 3$ and $b<1+d\left(\frac{a-1}{a}\right)$ ( or $\frac{b-1}{d}<\frac{a-1}{a}$ ), then $\left(\frac{a-1}{a}, 0\right)$ is asymptotically stable and species $y$ goes extinct. B - If $1<b \leq 3$ and $b>\frac{c}{1+c-a}$ ( or $\frac{b-1}{b}>\frac{a-1}{c}$ ), then $\left(0, \frac{b-1}{b}\right)$ is asymptotically stable and species $x$ goes extinct.

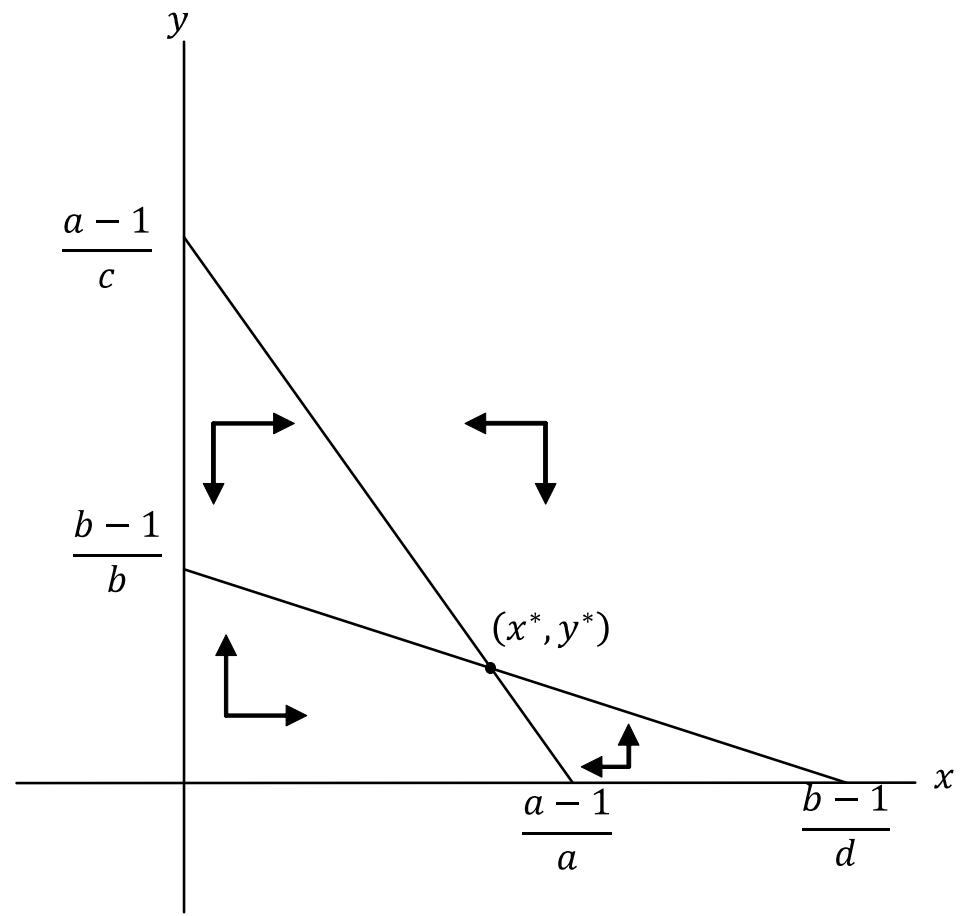

Figure 7. Isoclines: a positive fixed point exists if $\frac{a-1}{c}>\frac{b-1}{b}$ and $\frac{b-1}{d}>\frac{a-1}{a}, a, b>1$ and $c, d \in(0,1)$.

with $a>1$ and $b>1$.

The Jacobian evaluated at the coexistence fixed point $\left(x^{*}, y^{*}\right)$ is given by

$$
J^{*}=J F\left(x^{*}, y^{*}\right)=\left[\begin{array}{cc}
\frac{1-2 x^{*}}{1-x^{*}} & \frac{-c x^{*}}{a\left(1-x^{*}\right)} \\
\frac{-d y^{*}}{b\left(1-y^{*}\right)} & \frac{1-2 y^{*}}{1-y^{*}}
\end{array}\right]
$$

Let $P(\lambda)$ be the characteristic polynomial of $J^{*}$. According to the Jury test the eigenvalues of $J^{*}$ lie inside the unit disk iff 
(i) $P(1)>0$, (ii) $P(-1)>0$ and (iii) $|P(0)|<1$.

Note that the existence of the coexistence fixed point is assured by relation (17). Here $a>1$ and $b>1$ which implies that $a b-c d>0$. We assume that both, $a$ and $b$ have an upper boundary, i.e.,

$$
1<a<3 \text { and } 1<b<3 .
$$

Assumption (18) implies that $x^{*}<\frac{2}{3}$ and $y^{*}<\frac{2}{3}$.

After some computation one obtains

$$
\begin{aligned}
P(1) & =\frac{[a(b-1)-d(a-1)] \cdot(a b-c d)}{a b} \frac{b(a-1)-c(b-1)}{[a(1+d)-d(1+c)](b(1+c)-c(1+d))} \\
& =\frac{\left[b-\left(1+d\left(\frac{a-1}{a}\right)\right)\right](a b-c d)\left[a-\left(1+c\left(\frac{b-1}{b}\right)\right)\right]}{[a(1+d)-d(1+c)][b(1+c)-c(1+c)]}
\end{aligned}
$$

witch is a positive number by (17). Thus $P(1)>0$.

We also obtain

$$
\begin{aligned}
P(-1) & =\left(\frac{1-2 x^{*}}{1-x^{*}}+1\right)\left(\frac{1-2 y^{*}}{1-y^{*}}+1\right)-\frac{c d}{a b} \frac{x^{*} y^{*}}{\left(1-x^{*}\right)\left(1-y^{*}\right)} \\
& =\frac{\left(2-3 x^{*}\right)\left(2-3 y^{*}\right)-\frac{c d}{a b} x^{*} y^{*}}{\left(1-x^{*}\right)\left(1-y^{*}\right)}
\end{aligned}
$$

By the fact that $\left(1-x^{*}\right)\left(1-y^{*}\right)>0$ it follows that $P(-1)>0$ if only if $\left(2-3 x^{*}\right)\left(2-3 y^{*}\right)-\frac{c d}{a b} x^{*} y^{*}>0$. But

$$
\left(2-3 x^{*}\right)\left(2-3 y^{*}\right)-\frac{c d}{a b} x^{*} y^{*}>0
$$

is equivalent to

$$
\left(x^{*}+y^{*}\right)-\frac{4}{3}<\left(3-\frac{c d}{3 a b}\right) x^{*} y^{*}
$$

which is true under hypothesis (18). Thus one has $P(-1)>0$.

Finally, we investigate the third relation $|P(0)|<1$ or equivalently $P(0)>-1$ and $P(0)<1$.

Computations shows that

$$
\begin{aligned}
P(0) & =\frac{a\left(2 x^{*}-1\right)}{1+c y^{*}} \frac{b\left(2 y^{*}-1\right)}{1+d y^{*}}-\frac{a b c d x^{*}\left(x^{*}-1\right) y\left(y^{*}-1\right)}{\left(1+c y^{*}\right)^{2}\left(1+d x^{*}\right)^{2}} \\
& =\frac{2 x^{*}-1}{1-x^{*}} \frac{2 y^{*}-1}{1-y^{*}}-\frac{c d x^{*} y^{*}}{\left(1+c y^{*}\right)\left(1+d x^{*}\right)} \\
& =\frac{\left(2 x^{*}-1\right)\left(2 y^{*}-1\right)}{\left(1-x^{*}\right)\left(1-y^{*}\right)}-\frac{\frac{c d}{a b} x^{*} y^{*}}{\left(1-x^{*}\right)\left(1-y^{*}\right)}=\frac{\left(2 x^{*}-1\right)\left(2 y^{*}-1\right)-\frac{c d}{a b} x^{*} y^{*}}{\left(1-x^{*}\right)\left(1-y^{*}\right)}
\end{aligned}
$$

Thus one has the following two inequalities

$$
-1<\frac{\left(2 x^{*}-1\right)\left(2 y^{*}-1\right)-\frac{c d}{a b} x^{*} y^{*}}{\left(1-x^{*}\right)\left(1-y^{*}\right)}<1 .
$$




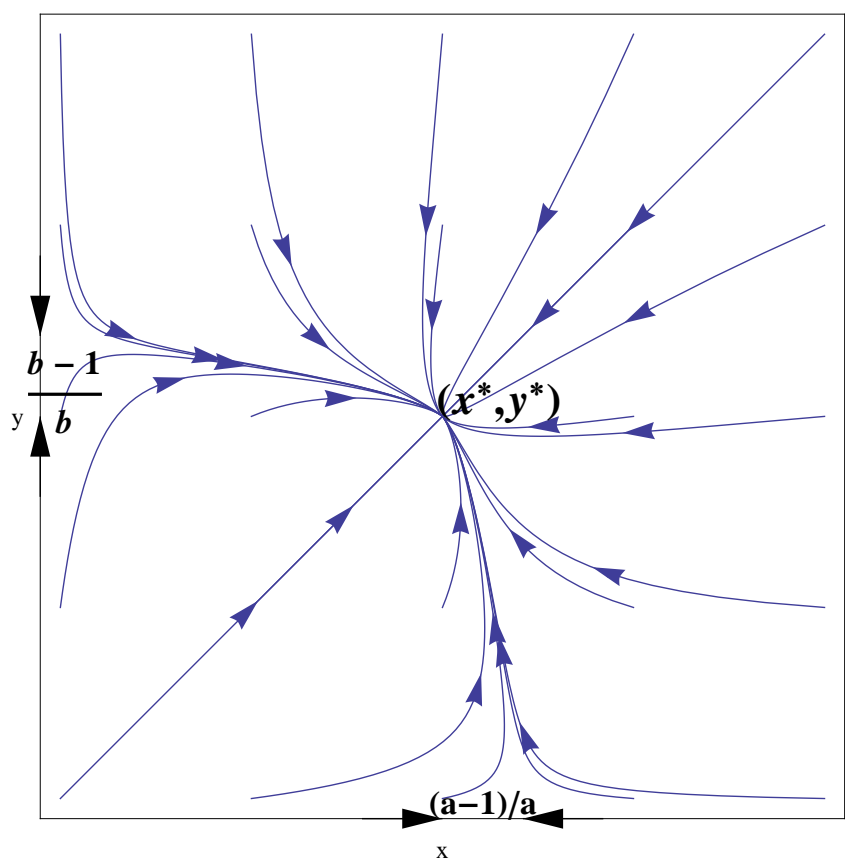

Figure 8. Phase-space diagram for the coexistence fixed point of the competition logistic model

The relation

$$
\frac{\left(2 x^{*}-1\right)\left(2 y^{*}-1\right)-\frac{c d}{a b} x^{*} y^{*}}{\left(1-x^{*}\right)\left(1-y^{*}\right)}+1>0
$$

is equivalent to

$$
\left(x^{*}+y^{*}\right)-\frac{2}{3}<\left(5-\frac{c d}{a b}\right) x^{*} y^{*}
$$

which is true under assumption (18).

The second inequality

$$
\frac{\left(2 x^{*}-1\right)\left(2 y^{*}-1\right)-\frac{c d}{a b} x^{*} y^{*}}{\left(1-x^{*}\right)\left(1-y^{*}\right)}-1<0
$$

is equivalent to

$$
\frac{1}{x^{*}}+\frac{1}{y^{*}}>3-\frac{c d}{a b}
$$

which is a true under hypothesis (18). Consequently, under hypothesis (18) the relation $|P(0)|<1$ is verified.

In conclusion, the coexistence fixed point $\left(x^{*}, y^{*}\right)$ is asymptotically stable if $1<$ $a<3$ and $1<b<3$. In Figure 8 we present the phase-space diagram when this coexistence fixed point is asymptotically stable.

In the next section we will show, through simulations, that the region of stability of the coexistence fixed point is indeed much larger than the square $1<a<3$ and $1<b<3$. 


\section{A bifurcation scenario}

The main objective in this section is to find, in the parameter space, the bifurcation scenario of the fixed point of the competition logistic map.

The region where the coexistence fixed point is asymptotically stable is given by the following relations [5, pp 200]

$$
\left|\operatorname{tr}\left(J^{*}\right)\right|-1<\operatorname{det}\left(J^{*}\right)<1,
$$

where $t r$ and det denote the trace and the determinant of the matrix, respectively. If at least one of these three inequalities is reversed, then the fixed point is unstable.

Our calculations show that

$$
\begin{aligned}
\operatorname{det}\left(J^{*}\right)= & \frac{-c(b-c+b c) d^{2}+a^{3} b^{2}(2-b+2 d)-a d\left(3 b c-b^{2}\left(4+5 c+c^{2}\right)+c^{2}(1+d)\right)}{(a b(-(1+c) d+a(1+d))(-b(1+c)+c(1+d)))} \\
& +\frac{a^{2} b\left(2 b^{2}(1+c)-b(4+6 c+6 d+5 c d)+c\left(4+5 d+d^{2}\right)\right)}{(a b(-(1+c) d+a(1+d))(-b(1+c)+c(1+d)))}
\end{aligned}
$$

and

$$
\begin{aligned}
\operatorname{tr}\left(J^{*}\right)= & \frac{a^{2} b(1+d)+d\left(b\left(4+7 c+3 c^{2}\right)-c(4+3 c+3 d+2 c d)\right)}{((1+c) d-a(1+d))(b(1+c)-c(1+d))} \\
& +\frac{a\left(b^{2}(1+c)-b(4+5 c+5 d+6 c d)+c\left(4+7 d+3 d^{2}\right)\right)}{((1+c) d-a(1+d))(b(1+c)-c(1+d))} .
\end{aligned}
$$

Thus, the inequality $\operatorname{det}\left(J^{*}\right)<1$ leads to

$$
\begin{aligned}
& \frac{-c(b-c+b c) d^{2}+a^{3} b^{2}(2-b+2 d)+a(b-c+b c) d(3 b+c+c d)}{a b(-(1+c) d+a(1+d))(-b(1+c)+c(1+d))} \\
& +\frac{a^{2} b\left(2 b^{2}(1+c)+3 c(1+d)-b(3+5 d+c(5+4 d))\right)}{a b(-(1+c) d+a(1+d))(-b(1+c)+c(1+d))}<0,
\end{aligned}
$$

the inequality $\operatorname{det}\left(J^{*}\right)>\operatorname{tr}\left(J^{*}\right)-1$ is equivalent to

$$
\frac{(b(-1+a-c)+c)(a(-1+b-d)+d)(a b-c d)}{a b(-(1+c) d+a(1+d))(-b(1+c)+c(1+d))}<0
$$

and the inequality $\operatorname{det}\left(J^{*}\right)>-\operatorname{tr}\left(J^{*}\right)-1$ is equivalent to

$$
\begin{aligned}
& \frac{-c(b-c+b c) d^{2}+a^{3} b^{2}(3-b+3 d)-a d\left(-b^{2}\left(9+14 c+5 c^{2}\right)+c^{2}(1+d)+b c(8+4 c+4 d+3 c d)\right)}{a b(-(1+c) d+a(1+d))(-b(1+c)+c(1+d))} \\
& +\frac{a^{2} b\left(3 b^{2}(1+c)+c\left(9+14 d+5 d^{2}\right)-3 b(3+4 d+4 c(1+d))\right)}{a b(-(1+c) d+a(1+d))(-b(1+c)+c(1+d))}>0 .
\end{aligned}
$$

Let $S_{1}$ be the set of points in the $(a, b)$-plane such that relations (20), (21) and (22) are satisfied. This is precisely the region in the parameter space where the coexistence fixed point is asymptotically stable. Zone $S_{1}$ is shown in Figure 9. Note that the parameters $c$ and $d$ are fixed.

In [5] the author presents a complete study of the three main types of bifurcation, for two-dimensional systems. The saddle-node bifurcation occurs when the 


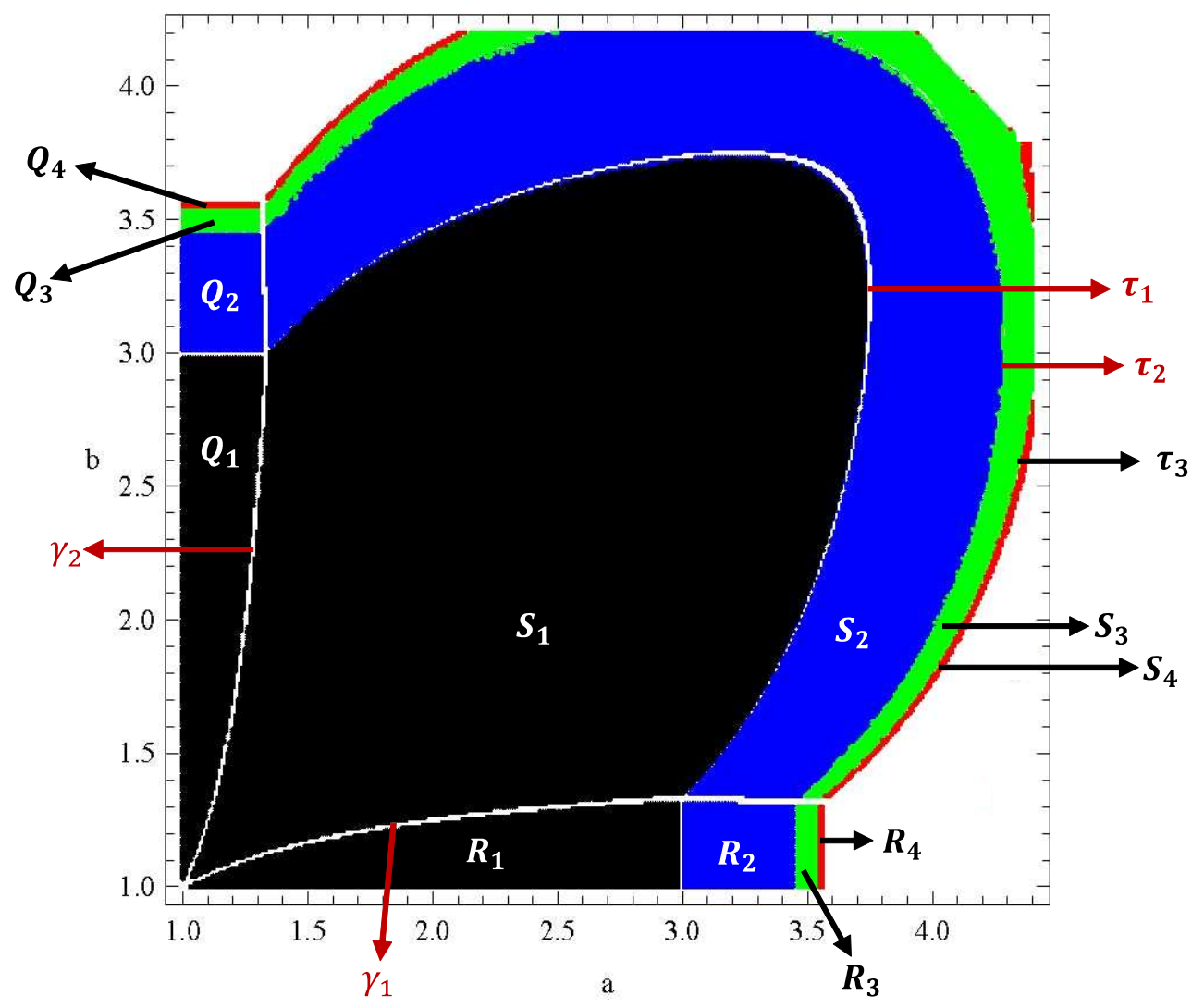

Figure 9. The bifurcation scenario of a fixed point of the competition logistic model in the parameter space $(a, b)$

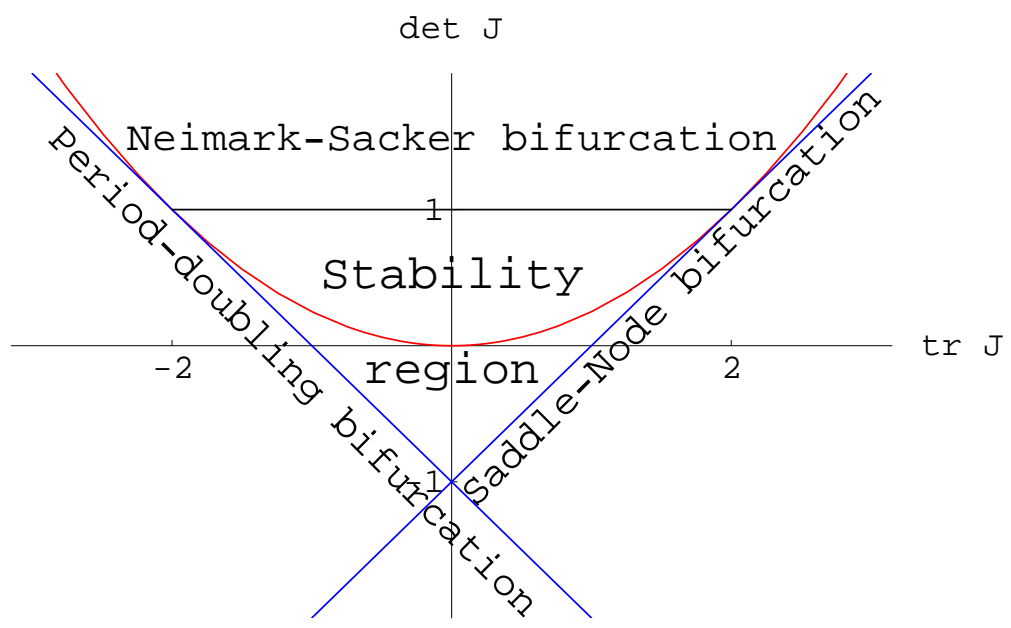

Figure 10. The occurrence of the three main types of bifurcation in the Trace-Determinant plane T-D

Jacobian has an eigenvalue equal to one. In the Trace-Determinant plane (T-D), this is equivalent to saying that we cross the line $\operatorname{det}\left(J^{*}\right)=\operatorname{tr}\left(J^{*}\right)-1$ from the stability region (see Figure 10). The period-doubling bifurcation occurs when the Jacobian has an eigenvalue equal to -1 . In the T-D plane this occurs as we cross the line $\operatorname{det}\left(J^{*}\right)=-\operatorname{tr}\left(J^{*}\right)-1$ from the stability region. When the Jacobian has a pair of complex conjugate eigenvalues of modulus 1, we have the Neimark-Sacker bifurcation. This happens in the T-D plane when $\operatorname{det}\left(J^{*}\right)=1$ and $-2<\operatorname{tr}\left(J^{*}\right)<2$.

Eq. (1) has a saddle-node bifurcation when relation (21) is an equality. This leads 
to the equations $b=1+d\left(\frac{a-1}{a}\right)$ and $a=1+c\left(\frac{b-1}{b}\right)$. Let

$$
\gamma_{1}=\left\{(a, b) \in \mathbb{R}_{+}^{2}: b=1+d\left(\frac{a-1}{a}\right)\right\} \text { and } \gamma_{2}=\left\{(a, b) \in \mathbb{R}_{+}^{2}: a=1+c\left(\frac{b-1}{b}\right)\right\} .
$$

See Figure 9. Hence, when $a$ and $b$ changes from region $S_{1}$ to region $R_{1}$ in Figure 9, the coexistence fixed point undergoes a saddle-node bifurcation into another fixed point. Computations show that this is an exclusion fixed point on the $x$-axis. Thus if we take $a$ and $b$ in region $R_{1}$, then Eq. (1) possesses an exclusion fixed point on the $x$-axis. Similarly, if $a$ and $b$ are in region $Q_{1}$.

Eq. (1) has a period-doubling bifurcation when we have equality in relation (22). This is represented by the curve $\tau_{1}$ in Figure 9. Consequently, as $a$ and $b$ pass the curve $\tau_{1}$ the coexistence fixed point undergoes a period-doubling bifurcation into a coexistence 2-periodic cycle. Thus in region $S_{2}$ Eq. (1) has one unstable fixed point and one asymptotically stable coexistence 2 -periodic cycle.

When $a$ and $b$ pass the curve $\gamma$ from region $S_{2}$ to region $R_{2}$, the coexistence 2 -periodic cycle bifurcates (saddle-node). Computations shows that this new 2 -periodic cycle is an exclusion cycle on the $x$-axis. Another period-doubling bifurcation appears in the exclusion fixed point if we move the parameters $a$ and $b$ from region $R_{1}$ to region $R_{2}$. Thus if the parameters $a$ and $b$ are in region $R_{2}$, Eq. (1) has an asymptotically stable exclusion 2 -periodic cycle on the $x$-axis. Analogous results may be obtained if the parameters are in region $Q_{2}$.

The coexistence 2 -periodic cycle undergoes a period-doubling bifurcation when the parameters pass the curve $\tau_{2}$. Thus in region $R_{3}$ this 2 -periodic cycle becomes unstable and a new asymptotically stable 4 -periodic cycle is born. This new cycle undergoes a saddle-node bifurcation into an asymptotically stable exclusion 4 -periodic cycle on the $x$-axis whenever the parameters change from region $R_{3}$ to region $S_{3}$. We also have a period-doubling bifurcation of the exclusion 2 -periodic cycle if we move the parameters from region $R_{2}$ to region $R_{3}$. Thus in region $R_{3}$, Eq. (1) has an asymptotically stable exclusion $4-$ periodic cycle (the same happens on the $y$-axis if the parameters changes from region $R_{3}$ to the region $Q_{3}$ ).

Note that the sequence of bifurcation points on the axes agree with the sequence of period doubling bifurcation parameters of the logistic map in one dimension.

This scenario of bifurcation continues in its route to chaos. Thus there exists a curve $\tau_{\infty}$ in the parameter space after which we enter a chaotic region. Moreover, there exists a curve after which all the iterations of Eq. (1) go to $(-\infty,-\infty)$. Note that on the axes $a$ and $b$ this corresponds to the value $a=4$ and $b=4$.

As a consequence of the scenario described above, one may conclude that, for fixed parameters $c$ and $d$, if the parameters $a$ and $b$ belong to the region $R_{i}$, $i=1,2, \ldots$, then species $y$ will go extinct. A similar behavior is exhibited by species $x$ if the parameters $a$ and $b$ are in region $Q_{i}, i=1,2, \ldots$ The coexistence of both species is possible whenever the parameters $a$ and $b$ are in the stability region $S_{i}, i=1,2, \ldots$

\section{References}

[1] X. Cabre, E. Fontich and R. de la LLave, The parameterization method for invariant manifolds: Manifolds associated to non-resonant subspaces, Manuscript, 1999

[2] J. Carr, Application of Center Manifolds Theory, Applied Mathematical Sciences, 35, Springer-Verlag, New York, 1981

[3] J. M. Cushing, S. LeVarge, N. Chitnis and S. M. Henson, Some discrete competition models and the competitive exclusion principle, Journal of Difference Equations and Applications, 10(13) (2004), pp. 1139-1151.

[4] Z. Di and M. Sanglier, A two-dimensional logistic model for the interaction of demand and supply and its bifurcations, Chaos Solutions and Fractals, 7(12)(1996), pp. 2259-66.

[5] S. Elaydi, Discrete Chaos: With Applications in Science and Engineering, Chapman and Hall/CRC, Second Edition, 2008. 
[6] J. Franke and A. Yakubu, Mutual exclusion versus coexistence for discrete competitive systems, J. Math. Biol., 30(2) (1991), pp. 161-168.

[7] P. Gomis-Porqueras and A. Haro, Global dynamics in macroeconomics: an overlapping generations example, Journal of Economic Dynamics \& Control, 27 (2003), pp. 1941-1959

[8] M. Hassell and H. Comins, Discrete time models for two-species competition, Theor. Popul. Biol., 9 (1976), pp. 202-221

[9] W. Hernandez-Padilla and L.-I. Roeger, Local stability of a discrete competition model derived from a nonstandard numerical method, Advances in Discrete Dynamical Systems, Proceedings of 11st ICDEA 2006, Vol. 53, Japan, 2009.

[10] G. Iooss, Bifurcation of maps and application, North-Holland Mathematics Studies, 1979.

[11] N. Karydas and J. Schinas, The center manifold theorem for a discrete system, Applicable Analysis, 44 (1992), pp. 267-284

[12] A. Kelly, The stable, center-stable, center-unstable and unstable manifolds, J. Differential Equations, 3 (1967), pp. 546-570

13] P. Leslie and J. Gower, The properties of a stochastic model for two competing species, Biometrika, 45 (1958), pp. 316-330.

[14] P. Liu and S. N. Elaydi, Discrete competitive and cooperative models of Lotka-Volterra type, Journal of Computational Analysis and Applications, 3 (2001), pp. 53-73.

[15] R. Lpez-Ruiz and D. Fournier-Prunaret, Complex behavior in a discrete coupled logistic model for the symbiotic interaction of two species, Math Biosci Eng, 1 (2004), pp. 307-324.

[16] J. Marsden and M. McCracken, The hopf bifurcation and its application, Springer-Verlag, New York, 1976.

[17] L.-I. Roeger, Discrete May-Leonard competition models III, Journal of Difference Equations and Applications, 10(8) (2004), pp. 773-790.

[18] W.Rudin, Principles of Mathematical Analysis, McGraw - Hill, Third Edition, 1976.

[19] G. Sorger, Discrete time dynamic game models for advertising competition in a duopoly, Optimal Control Applications and Methods, 16 (1995), pp. 175-188.

[20] M. Succi, S. Lee and J. Alexander, Effects of Market Position and Competition on Rural Hospital Closures, Health Services Research, 31 (1997), pp. 679-99.

[21] A. Vanderbauwhede, Center Manifolds, Normal Forms and Elementary Bifurcations, In Dynamics Reported, Vol. 2 (1989)

[22] S. Wiggins, Introduction to Applied Nonlinear Dynamical Systems and Chaos, Springer, 2003

\section{Appendix A. Values of the coefficients $p_{i}, i=1, \ldots, 14$ (stability of the exclusion fixed point when $a=3$ )}

$$
\begin{gathered}
p_{1}=81 \alpha-81 b \alpha+54 d \alpha \\
p_{2}=486 \alpha+405 d \alpha+108 c \alpha^{2}+72 c d \alpha^{2}-81 \beta-81 b \beta-54 d \beta
\end{gathered}
$$

$p_{3}=729 \alpha+972 d \alpha+81 b \alpha^{2}+405 c \alpha^{2}-243 b c \alpha^{2}+378 c d \alpha^{2}+36 c^{2} \alpha^{3}+24 c^{2} d \alpha^{3}-$

$729 \beta-567 d \beta-54 c \alpha \beta-36 c d \alpha \beta$,

$$
\begin{aligned}
p_{4}= & 729 d \alpha+486 c \alpha^{2}+729 c d \alpha^{2}+108 c^{2} \alpha^{3}+108 c^{2} d \alpha^{3}-2187 \beta-2187 d \beta+162 b \alpha \beta- \\
& 567 c \alpha \beta-486 b c \alpha \beta-432 c d \alpha \beta-36 c^{2} \alpha^{2} \beta-24 c^{2} d \alpha^{2} \beta-162 c \beta^{2}-108 c d \beta^{2},
\end{aligned}
$$

$$
\begin{aligned}
p_{5}= & 729 c \alpha^{2}+972 c d \alpha^{2}+243 b c \alpha^{3}+324 c^{2} \alpha^{3}-243 b c^{2} \alpha^{3}+324 c^{2} d \alpha^{3}+36 c^{3} \alpha^{4}+ \\
& 24 c^{3} d \alpha^{4}-2187 \beta-3645 d \beta-972 c \alpha \beta-1215 c d \alpha \beta-108 c^{2} \alpha^{2} \beta-108 c^{2} d \alpha^{2} \beta- \\
& 24 c^{3} \alpha^{3} \beta-16 c^{3} d \alpha^{3} \beta+81 b \beta^{2}-972 c \beta^{2}-243 b c \beta^{2}-810 c d \beta^{2}-180 c^{2} \alpha \beta^{2}- \\
& 120 c^{2} d \alpha \beta^{2},
\end{aligned}
$$




$$
\begin{gathered}
p_{6}=729 c d \alpha^{2}+324 c^{2} d \alpha^{3}+36 c^{3} d \alpha^{4}-2187 d \beta+729 c \alpha \beta-486 c d \alpha \beta+729 b c \alpha^{2} \beta+ \\
648 c^{2} \alpha^{2} \beta-729 b c^{2} \alpha^{2} \beta+324 c^{2} d \alpha^{2} \beta+108 c^{3} \alpha^{3} \beta+48 c^{3} d \alpha^{3} \beta-1458 c \beta^{2}- \\
1944 c d \beta^{2}-540 c^{2} \alpha \beta^{2}-540 c^{2} d \alpha \beta^{2}-72 c^{3} \alpha^{2} \beta^{2}-48 c^{3} d \alpha^{2} \beta^{2}-108 c^{2} \beta^{3}-72 c^{2} d \beta^{3}, \\
p_{7}=243 b c^{2} \alpha^{4}-81 b c^{3} \alpha^{4}+729 c d \alpha \beta+648 c^{2} d \alpha^{2} \beta+108 c^{3} d \alpha^{3} \beta-1458 c d \beta^{2}+ \\
729 b c \alpha \beta^{2}+324 c^{2} \alpha \beta^{2}-729 b c^{2} \alpha \beta^{2}-324 c^{2} d \alpha \beta^{2}+108 c^{3} \alpha^{2} \beta^{2}-324 c^{2} \beta^{3}- \\
324 c^{2} d \beta^{3}-72 c^{3} \alpha \beta^{3}-48 c^{3} d \alpha \beta^{3}, \\
p_{8}=972 b c^{2} \alpha^{3} \beta-324 b c^{3} \alpha^{3} \beta+324 c^{2} d \alpha \beta^{2}+108 c^{3} d \alpha^{2} \beta^{2}+243 b c \beta^{3}-243 b c^{2} \beta^{3}- \\
324 c^{2} d \beta^{3}+36 c^{3} \alpha \beta^{3}-48 c^{3} d \alpha \beta^{3}-24 c^{3} \beta^{4}-16 c^{3} d \beta^{4}, \\
p_{9}=81 b c^{3} \alpha^{5}+1458 b c^{2} \alpha^{2} \beta^{2}-486 b c^{3} \alpha^{2} \beta^{2}+36 c^{3} d \alpha \beta^{3}-24 c^{3} d \beta^{4}, \\
p_{10}=405 b c^{3} \alpha^{4} \beta+972 b c^{2} \alpha \beta^{3}-324 b c^{3} \alpha \beta^{3}, \\
p_{11}=810 b c^{3} \alpha^{3} \beta^{2}+243 b c^{2} \beta^{4}-81 b c^{3} \beta^{4}, \\
p_{12}=810 b c^{3} \alpha^{2} \beta^{3}, \\
p_{13}=405 b c^{3} \alpha \beta^{4},
\end{gathered}
$$

and

$$
p_{14}=81 b c^{3} \beta^{5} .
$$

